\title{
Fabrication of cylindrical resonant microcavities using the confinement provided by partially oxidized AIGaAs/GaAs layers
}

\author{
Pedro V. Pinho N.*, Gustavo A. Palomino M., Ailton J. Garcia Jr., Christoph F. Deneke, Newton C. Frateschi.
}

\begin{abstract}
Two different interfaces for optical confinement in GaAs resonators (air/GaAs/air, air/GaAs/AlOx) are compared in cylindrical microstructures with $10 \mu \mathrm{m}$ diameter. The AIOx is obtained by the lateral oxidation of AIGaAs layers. The spectrum of the resonators is obtained using a tapered fiber system in the wavelength range of $1470 \mathrm{~nm}$ to $1610 \mathrm{~nm}$. The modal separation agrees with our simulation. The quality factor for cylindrical microstructures with air/GaAs/air interface is almost twice the case with AlOx interface. Since it is expected that surface recombination velocity is reduced by a factor of ten for this last case, these structures are shown to be suitable for active resonators.
\end{abstract}

\section{Key words:}

oxidation of $A / G a A s$, whispering gallery modes, quality factor

\section{Introduction}

It is known that GaAs-based active devices are prone to nonradiative surface recombination at unpassivated surfaces. In GaAs cylindrical microresonators, the employment of passivation technique enhances maximal temperature operation and output power. In addition, a reduction of threshold power is observed. A selective oxidation of $\mathrm{AlGaAs}$ cladding layers has been proposed by Holoyank and Dallese. The result of the oxidation in the first instance is aluminum oxide (Al2O3) that provides chemical stability to the AIGaAs layer. Aluminum oxide is an insulating material that reduces the refractive index by half, $n(A I G a A s)=3$ and $n A I O x=1.5$. These characteristics are used for the confinement of electron and photon in the devices. In this work, we performed the characterization of AIGaAs oxidation process on $500 \mathrm{~nm}$ and $300 \mathrm{~nm}$ thickness samples. Then two types of cylindrical resonators for optical confinement in GaAs layer were fabricated based on the oxidation results. The difference between them is the interface for GaAs layer: air/GaAs/air and air/GaAs/AIOx. The spectrum of the resonators were obtained using a tapered fiber system in the wavelength range of $1470 \mathrm{~nm}$ to $1610 \mathrm{~nm}$ and compared with simulation results.

\section{Results and Discussion}

At $380^{\circ} \mathrm{C}$ the growth rate oxide was of $0.0125 \mu \mathrm{m} / \mathrm{min}$ without $\mathrm{GaAs}$ layer degradation for the cylindrical mesas of the samples used. The Fig. 1 shows the depth oxide with time at $380^{\circ} \mathrm{C}$ on the samples. It is important to note that the length oxide is linear to time and is have not dependence with of the samples thickness. Fig. 2 shows the measure spectrum of microresonator with air/GaAs/air interface in the range from $1470 \mathrm{~nm}$ to 1590 $\mathrm{nm}$ plotted with a blue line and the spectrum obtained solving the transcendental equation is plotted in bars. The amplitude of the bars was placed to better identify the modes in the measured spectrum. Comparing simulation and experimental results of the microresonator spectrum is observed a better propagation of the TE modes of second radial order. The maximum error between measure and simulation is $4 \mathrm{~nm}$ because the mode at $1497 \mathrm{~nm}$ is not clearly defined. The propagation of second radial order modes may occurred because the fiber diameter was almost the same length as the confinement region. This did not allow the propagation of WGM, and besides, was not big enough to excite the propagation of higher radial order modes. Fig. 3 shows a measure spectrum obtained in the range from $1464 \mathrm{~nm}$ to $1560 \mathrm{~nm}$ plotted with a blue line for microresonators with air/GaAs/AlOx interface. The maximum error between measure and simulation results is $4 \mathrm{~nm}$ at $1480 \mathrm{~nm}$. Similarly to previous result, TE modes of second radial order propagates better.
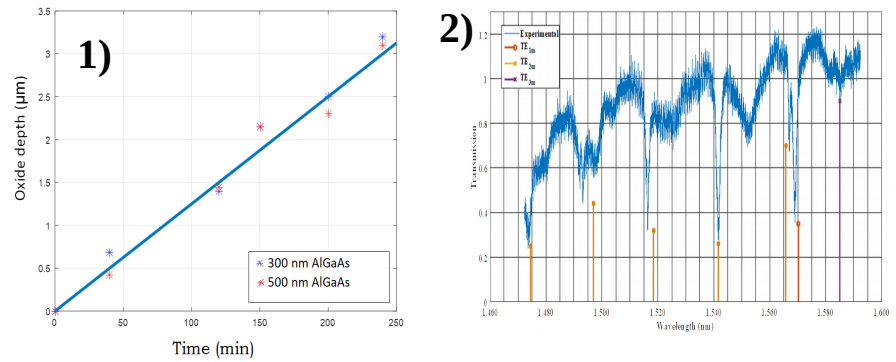

3)

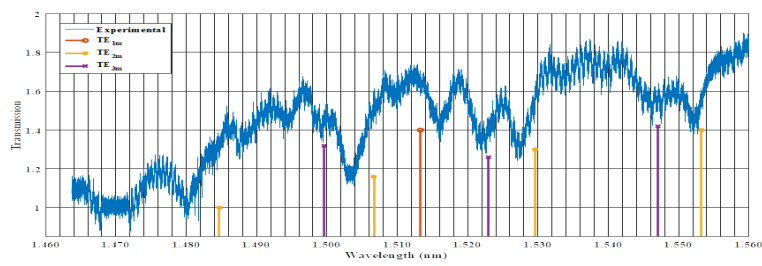

\section{Conclusions}

We have fabricated GaAs microresonators using lateral oxidation of $\mathrm{AIGaAs}$ layers at $380^{\circ} \mathrm{C}$ without GaAs layer degradation. Microresonators with air/GaAs/air interface shown better spectrum features than air/GaAs/AIOx interface resulting in better agreement with simulations. The quality factor for cylindrical microstructures with air/GaAs/air interface is almost twice the case with AIOx interface. Since it is expected that surface recombination velocity is reduced by a factor of ten for this last case, these structures are shown to be suitable for active resonators.

\section{Acknowledgement}

We would like to acknowledge financial support from FAPESP under Grant (2017/09481-2), FAPESP under the contract 2015/24517-8 and CNPq: 305907/2018-2. 